\title{
Analysis of Rigid Body Interactions for Compliant Motion Tasks Using the Grassmann-Cayley Algebra
}

\author{
E. Staffetti F. Thomas \\ Institut de Robòtica i Informàtica Industrial (UPC-CSIC) \\ Gran Capità 2-4, 08034 Barcelona, Spain \\ \{estaffetti, fthomas\}@iri.upc.es
}

\begin{abstract}
This paper studies the statics and the instantaneous kinematics of a rigid body constrained to keep an arbitrary number of surface-surface contacts with a rigid static environment during its motion.

These properties are analyzed under the frictionless assumption by modelling each contact with a kinematic chain that instantaneously gives the same motion freedom as the contact itself and by studying the resulting parallel chain using the Grassmann-Cayley Algebra.

With this algebra twists and wrenches can be expressed by means of extensors and operated using the join and meet operators. Moreover, the duality inherent in this algebra is used to reflect the reciprocity condition between possible twists and admissible wrenches between partially constrained rigid bodies.
\end{abstract}

\section{Introduction}

Compliant motion tasks are manipulation tasks that involve contacts between the manipulated object, say $M$, and the static environment, say $S$, in which the trajectory of the manipulator is modified depending on the contact forces arising between them.

In these applications it is important to know how $S$ constrains the motion of $M$ and the possible reaction forces that might arise in their interaction in order to appropriately choose the generalized motions and forces to accomplish a given task.

In this paper we shall study the kinestatics of a partially constrained rigid body, that is, the statics and instantaneous kinematics of a rigid object constrained to keep an arbitrary number of surface-surface contacts with a rigid static environment, thus extending the work presented in [1] in which some aspects of the same problem were studied using the Grassmann-Cayley
Algebra on polyhedral approximations of the objects involved in the motion.

The precision in the execution of a compliant task relies on the accuracy of the model but exact modelling of the motion constraints could be impractical. So, with the hypothesis of the absence of friction, we use a second order local approximation that takes into account not only the tangent plane at the contact point but also the principal directions and the principal curvatures of the surface patches at that point, which are easily computable from both parametric and implicit representations of surfaces $[2,3]$.

If each contact between $M$ and $S$ is translated into a kinematic chain that instantaneously gives the same motion freedom, a parallel mechanism is obtained whose instantaneous mobility coincides with that of $M$ with reference to $S$. This model has been introduced in [4] where the kinematic chains that represent the surface-surface contacts are called virtual contact manipulators and the effects of multiple contacts are studied by means of their Jacobian matrices.

The parallel mechanism resulting from translating each contact into its instantaneously equivalent kinematic chain is herein described and analyzed using the Grassmann-Cayley Algebra focusing the attention on the relationship between possible twists and admissible wrenches of the body that represents the moving object. In this way the results on the kinestatics of serial and parallel manipulators [5] can be applied to the analysis of rigid body interaction.

A comprehensive reference on the abstract properties of the Grassmann-Cayley Algebra can be found in [6] while $[7,8,9]$ emphasize the concrete approach to this algebra and give more detail on the connections to Robotics. This algebra is briefly reviewed in Sect. 2 .

Any instantaneous motion of a rigid body can be described as a twist on a screw and any set of forces and moments that act on a rigid body can be described as a wrench on a screw $[10,11]$. Sect. 3 describes 
how instantaneous screws can be represented by means of the concept of extensor of the Grassmann-Cayley Algebra.

Moreover, the twist and wrench spaces resulting from serial or parallel connections of motion constraints can be computed by means of the join and meet operators of this algebra and its inherent duality can be used to reflect the reciprocity between twists and wrenches of partially constrained rigid bodies. This is discussed in Sect. 4.

Sect. 5 presents the adopted equivalent kinematic model of a point surface-surface contact and in Sect. 6 the kinestatic analysis of the interaction between two rigid bodies is reported.

An example in which these concepts are applied to the study of the possible twists and admissible wrenches for a moving body that makes three point contacts with a static environment is presented in Sect. 7. Finally, Sect. 8 contains the conclusions.

\section{The Grassmann-Cayley Algebra}

In this section we report the most relevant properties of a version of the Grassmann-Cayley Algebra that involves Plücker coordinates. Further details can be found in [8].

It is convenient to start from some basic concept about projective space and homogeneous coordinates. If $\mathbf{x}=\left(x_{1}, x_{2}, \ldots, x_{m}\right)^{t}$ is a point in $\Re^{m}$ given in terms of Cartesian coordinates, the vector $x=$ $\left(x_{1}, x_{2}, \ldots, x_{m}, 1\right)^{t}$ is defined to be its homogeneous coordinate vector.

If we allow points with the last coordinate 0 for representing projective points at infinity, the standard $m$-dimensional projective space that includes $\Re^{m}$ is obtained. So, we represent $\Re^{m}$ with points at infinity by a $(m+1)$-dimensional vector space $V$.

\subsection{Plücker Coordinates}

Let $U$ be a $k$-dimensional subspace of the $(m+1)$ dimensional vector space $V$, and $\left\{u_{1}, u_{2}, \ldots, u_{k}\right\}$ a basis of it. When these vectors are arranged as rows of a matrix, we obtain:

$$
\left(\begin{array}{ccccc}
u_{1,1} & u_{1,2} & \cdots & u_{1, m} & 1 \\
u_{2,1} & u_{2,2} & \cdots & u_{2, m} & 1 \\
\cdots & \cdots & \cdots & \cdots & \cdots \\
u_{k, 1} & u_{k, 2} & \cdots & u_{k, m} & 1
\end{array}\right) .
$$

The $\left(j_{1}, j_{2}, \cdots, j_{k}\right)$-th Plücker coordinate of the subspace $U$, denoted by $P_{j_{1}, j_{2}, \cdots, j_{k}}$, is the $k \times k$ determinant formed by the $k$ columns of the above matrix with indices $j_{1}, j_{2}, \cdots, j_{k}$. Since we have a
Plücker coordinate for each combination of the $k$ columns, the total number of Plücker coordinates is $\left(\begin{array}{c}m+1 \\ k\end{array}\right)$. The Plücker coordinate vector of the subspace $U$ is the vector $P_{U}$ that contains in some predetermined order its Plücker coordinates. $U$ uniquely determines $P_{U}$ up to a scalar multiple.

The Plücker coordinate vector of the line $\Lambda$ passing through points $a$ and $b$, represented in homogeneous coordinates by the rows of

$$
\left(\begin{array}{llll}
a_{1} & a_{2} & a_{3} & 1 \\
b_{1} & b_{2} & b_{3} & 1
\end{array}\right)
$$

is defined as:

$$
\begin{gathered}
P_{\Lambda}=\left(-P_{1,4},-P_{2,4},-P_{3,4}, P_{2,3},-P_{1,3}, P_{1,2}\right)^{t}= \\
\left(b_{1}-a_{1}, b_{2}-a_{2}, b_{3}-a_{3}, a_{2} b_{3}-a_{3} b_{2}, a_{3} b_{1}-a_{1} b_{3}, a_{1} b_{2}-a_{2} b_{1}\right)^{t}=
\end{gathered}
$$$$
(\mathbf{S}, \mathbf{r} \times \mathbf{S})^{t}
$$

where $\mathbf{S}=\mathbf{b}-\mathbf{a},(\mathbf{r} \times \mathbf{S})$ represents the moment of $\Lambda$ with respect to the origin, and $\mathbf{r}$ is any point on the line. The vector $P_{\Lambda}$ is determined by $\Lambda$ up to a scalar multiple. It is convenient to normalize it such that $\mathbf{S} \cdot \mathbf{S}=1$ as it will be used to represent rotations.

The point at infinity on $\Lambda$ has homogeneous coordinates $\left(b_{1}-a_{1}, b_{2}-a_{2}, b_{3}-a_{3}, 0\right)^{t}$ and can be thought as infinitely far away in the direction given by S.

A line at infinity is determined by two distinct points, $c$ and $d$, at infinity represented by the rows of

$$
\left(\begin{array}{llll}
c_{1} & c_{2} & c_{3} & 0 \\
d_{1} & d_{2} & d_{3} & 0
\end{array}\right)
$$

whose Plücker coordinate vector is

$$
\begin{array}{r}
P_{\Lambda}=\left(0,0,0, c_{2} d_{3}-c_{3} d_{2}, c_{3} d_{1}-c_{1} d_{3}, c_{1} d_{2}-c_{2} d_{1}\right)^{t}= \\
(0,0,0, \mathbf{r} \times \mathbf{S})^{t} .
\end{array}
$$

Since lines at infinity will be used to represent translations, it is convenient to normalize this vector in such a way that $(\mathbf{r} \times \mathbf{S}) \cdot(\mathbf{r} \times \mathbf{S})=1$.

The Plücker coordinate vector of the plane $\Pi$, determined by three finite points whose homogeneous coordinates are the rows of

$$
\left(\begin{array}{llll}
a_{1} & a_{2} & a_{3} & 1 \\
b_{1} & b_{2} & b_{3} & 1 \\
p_{1} & p_{2} & p_{3} & 1
\end{array}\right),
$$

is defined as

$$
P_{\Pi}=\left(P_{2,3,4},-P_{1,3,4}, P_{1,2,4}, P_{1,2,3}\right)^{t}=(\mathbf{N}, \mathbf{r} \cdot \mathbf{N})^{t},
$$

where $\mathbf{N}$ is a normal vector to $\Pi$ and $\mathbf{r}$ is the position vector of any point on $\Pi$. 


\subsection{Dual Plücker Coordinates}

Let $U$ be a $k$-dimensional subspace of the $(m+1)$ dimensional vector space $V$ and $\left\{u_{1}, u_{2}, \ldots, u_{k}\right\}$ a basis of it. We can construct the dual of the vector subspace $U$ as follows. Consider the linear system of equations

$$
\sum_{j=0}^{m+1} x_{j} u_{h, j}=0, h=1,2, \ldots, k .
$$

Since its matrix has rank $k$ its solution space is a $(m+1-k)$-dimensional vector space $U^{*}$. Let $\left\{w_{1}, w_{2}, \ldots, w_{m+1-k}\right\}$ be a basis of $U^{*}$. Then, we have the following relationships between the basis vectors of $U$ and $U^{*}$

$\sum_{j=0}^{m+1} w_{i, j} u_{h, j}=0, i=1,2, \ldots,(m+1-k), h=1,2, \ldots, k$

by which we can compute the basis of $U^{*}$ from the basis of $U$ up to a scalar multiple. The vector spaces $U$ and $U^{*}$ are said to be dual spaces. Thus, the dual Plücker coordinate vector of $U$ is defined to be the vector that contains the Plücker coordinates of $U^{*}$, called dual Plücker coordinates of $U$.

It can be proved that the number of dual Plücker coordinates equals the number of Plücker coordinates, the only difference between them being their ordering and some sign changes. For example, the Plücker coordinate vector of a line in $\Re^{3}$ has the first three and the last three elements interchanged with respect to the dual Plücker coordinate vector of the same line.

If we replace vectors of $V$ by hyperplanes we obtain its dual vector space $V^{*}$.

\subsection{The Join and Meet Operators}

Let $V$ be a $n$-dimensional vector space over the field $\Re, U$ a $k$-dimensional subspace of $V$, and $\left\{u_{1}, u_{2}, \ldots, u_{k}\right\}$ a basis of it. Let also $P$ be the Plücker coordinate vector of $U$, that is, a vector in the $\left(\begin{array}{l}n \\ k\end{array}\right)$ dimensional vector space $V^{(k)}$. The vector $P$ is called $k$-extensor or decomposable $k$-tensor and is denoted by

$$
P=\vee\left(u_{1} u_{2} \cdots u_{k}\right)=u_{1} \vee u_{2} \vee \cdots \vee u_{k} .
$$

The vector space $V^{(k)}$ also contains indecomposable $k$-tensors which can always be rewritten as linear combination of $k$-extensors.

The subspace $U$, also denoted by $\tilde{P}$, is defined as the support of $P$ and the scalar $k$ is defined to be the step of the extensor. Two $k$-extensors are equal up to a scalar multiple if, and only if, they have the same support.
Let $A=a_{1} \vee a_{2} \vee \cdots \vee a_{k}$ and $B=b_{1} \vee b_{2} \vee \cdots \vee b_{j}$ be two extensors. The join of $A$ and $B$ is defined as the $(j+k)$-extensor

$$
A \vee B=a_{1} \vee a_{2} \vee \cdots \vee a_{k} \vee b_{1} \vee b_{2} \vee \cdots \vee b_{j} .
$$

If the vectors $\left\{a_{1}, a_{2}, \ldots, a_{k}, b_{1}, b_{2}, \ldots, b_{j}\right\}$ are linearly dependent, then $A \vee B=0$. If they are independent,

$$
\overline{A \vee B}=\bar{A}+\bar{B}=\operatorname{span}(\bar{A} \cup \bar{B}) .
$$

This means that the join of two extensors represent the operation of joining the associated vector subspaces.

Now, another operation that plays a similar role for the intersection of subspaces is defined. Let $A$ and $B$ be the above two extensors with $(k+j) \geq n$. The meet of $A$ and $B$ is defined as:

$$
\begin{array}{r}
A \wedge B=\sum_{\sigma} \operatorname{sgn}(\sigma)\left[a_{\sigma(1)}, \ldots, a_{\sigma(n-j)}, b_{1}, \ldots, b_{j}\right] \\
a_{\sigma(n-j+1)} \vee a_{\sigma(n-j+2)} \vee \cdots \vee a_{\sigma(k)}
\end{array}
$$

where the brackets stand for determinants and the sum is taken over all the permutations $\sigma$ of $\{1,2, \ldots, k\}$ such that $\sigma(1)<\sigma(2)<\cdots<\sigma(n-j)$ and $\sigma(n-$ $j+1)<\sigma(n-j+2)<\cdots<\sigma(k)$. This formula, called the shuffle formula, is a useful tool in practical applications.

It can be proved that if $A \neq 0$ and $B \neq 0$ and $\bar{A} \cup \bar{B}$ spans $V$, then

$$
\overline{A \wedge \vec{B}}=\bar{A} \cap \bar{B} .
$$

An important property is that $\vee$ and $\wedge$ are dual operators. If we interchange $\vee$ and $\wedge$ we must interchange $V^{(k)}$ with $V^{*(n-k)}$.

The vector spaces $V^{(k)}$ can be combined into another vector space over $\Re$,

$$
\Lambda(V)=V^{(0)} \oplus V^{(1)} \oplus \cdots \oplus V^{(n)}
$$

where $V^{(0)}$ and $V^{(n)}$ both coincide with $\Re$. The elements of $\Lambda(V)$ are all tensors, that is, arbitrary linear combinations of extensors of various steps. We have that $\operatorname{dim}(\Lambda(V))=\sum_{k=0}^{n}\left(\begin{array}{l}n \\ k\end{array}\right)=2^{n}$.

The Grassmann-Cayley Algebra is defined as the vector space $\Lambda(V)$ with the operations $\vee$ and $\Lambda$. These operations are both associative, distributive over addition, and anticommutative.

\section{Projective Representation of Twists and Wrenches}

If $\mathbf{v}$ is the velocity of the Euclidean point $\mathbf{p}$, then the motion of the projective point $p$ can be defined as $M(p)=(\mathbf{v}, \mathbf{p} \cdot \mathbf{v})^{t}$. 
If $a$ and $b$ are finite projective points, for each point $p$ in space we can express this motion in projective terms as $M(p)=(\mathbf{v}, \mathbf{p} \cdot \mathbf{v})^{t}=a \vee b \vee p$. The 2-extensor $a \vee b$, that represents the line passing through $a$ and $b$, is called the center of the motion. Since $M(a)=0$ and $M(b)=0$, it can be used to represent a rotation about the axis determined by $a$ and $b$.

A translation can be described as a rotation about an axis at infinity. Let $c=\left(c_{1}, c_{2}, c_{3}, 0\right)^{t}$ and $d=\left(d_{1}, d_{2}, d_{3}, 0\right)^{t}$ be two points at infinity, then the extensor $c \vee d$ can be used as the center of motion such that $M(p)=c \vee d \vee p$. The corresponding velocity is $\mathbf{v}=\left(c_{2} d_{3}-c_{3} d_{2}, c_{3} d_{1}-c_{1} d_{3}, c_{1} d_{2}-c_{2} d_{1}\right)^{t}$. Since it is independent from the point $p$, it can be effectively used to represent a translation.

With a serial composition of translations and rotations a more general screw motion in space can be obtained. This motion is represented by a twist $t=$ $(\boldsymbol{\omega}, \mathbf{v})^{t}$, where $\boldsymbol{\omega}$ and $\mathbf{v}$ represent an angular velocity vector and a linear velocity vector, respectively. In general, a twist cannot be represented using the join of two projective points. Indeed it is an indecomposable 2-tensor that can be expressed as linear combination of 2-extensors. Moreover, according to the Chasles's theorem it can be expressed as the composition of a translation along an axis and a rotation about the same axis. Instantaneously, the serial composition of twists corresponds to their addition.

A vector $\mathbf{f}=\left(f_{1}, f_{2}, f_{3}\right)^{t}$ that represents an Euclidean force applied at the Euclidean point $\mathbf{p}=$ $\left(p_{1}, p_{2}, p_{3}\right)^{t}$ can be represented in the projective space by the join of the two projective points $p=$ $\left(p_{1}, p_{2}, p_{3}, 1\right)^{t}$ and $f=\left(f_{1}, f_{2}, f_{3}, 0\right)^{t}$, that is, by the extensor $F=p \vee f=(\mathbf{f}, \mathbf{p} \times \mathbf{f})^{t}$. If we have a set of forces $F_{i}=p \vee f_{i}$ applied at a given point $p$, the resultant, $\mathbf{G}$, can be obtained by adding the free vectors $f_{i}$ and applying the result at $p$, that is, $G=\sum_{i}\left(p \vee f_{i}\right)=p \vee\left(\sum_{i} f_{i}\right)$.

If two forces $F_{1}=p \vee f$ and $F_{2}=q \vee g$ with $\mathbf{f}=-\mathbf{g}$ are applied to two distinct points $p$ and $q$, the resultant $G=F_{1}+F_{2}=p \vee f+q \vee g=$ $p \vee f+q \vee(-f)=(p-q) \vee f$ is called a couple. Since $p-q=\left(p_{1}-q_{1}, p_{2}-q_{2}, p_{3}-q_{3}, 0\right)^{t}$ is a point at infinity a couple can be thought as a force at infinity.

In general, the composition of forces in space does not correspond to a single new force and cannot be expressed using the extensor of two projective points. Their resultant is a wrench $w=(\mathbf{f}, \mathbf{m})^{t}$, where $\mathbf{f}$ and $\mathbf{m}$ are a force vector and a moment vector, respectively. In general a wrench is an indecomposable 2 -tensor which can be expressed in terms of 2-extensor. Moreover, according to Poinsot's theorem a wrench can always be rewritten as a composition of a force along a line and a couple on the plane normal to the line.

Twists will be represented as linear combinations of 2-extensors of $V^{(2)}$ while wrenches will be represented in terms of the 2-extensors of its dual space $V^{*(2)}$.

Instantaneously, the resultant of the parallel composition of wrenches corresponds to their addition.

\section{Reciprocity}

Let us consider our rigid body $M$ partially constrained by the static environment $S$. We define the twist space $\mathbf{T}$ of $M$ as the vector space of all possible instantaneous twists that it can have with reference to $S$, and we define its wrench space $\mathbf{W}$ as the vector space of all reaction wrenches that can be generated in the interaction between $M$ and $S$.

Since the set of established contacts between $M$ and $S$ cannon be broken, any twist $t \in \mathbf{T}$ of $M$ must be reciprocal to any wrench $w \in \mathbf{W}$ between $M$ and $S$ because the power generated by $t$ and $w$ must be zero (see [12]), that is,

$$
\boldsymbol{\omega} \cdot \mathbf{m}+\mathbf{v} \cdot \mathbf{f}=0 .
$$

This relation is nothing more than the expression of the pairing of the vector spaces $V^{(2)}$ and its dual $V^{*(2)}$ as expressed by (1). Thus, we can say that twist and wrench spaces of a partially constrained rigid body are dual spaces in the sense of Sect. 2.2.

If the twist space of $M$ is the entire $V^{(2)}$, it has full mobility while, if its wrench space is the whole $V^{*(2)}$, it can apply any wrench to $S$. In general, since the twist space is a subspace of $V^{(2)}$ and the wrench space is a subspace of $V^{*(2)}$, it is convenient to set $V^{(2)}=H$ and work in the Grassmann-Cayley Algebra $\Lambda(H)$ over this auxiliary vector space in which 2-extensors of $V^{(2)}$ become 6-dimensional vectors.

It is well known (see, for example, [4]) that the twist (wrench) space of the serial (parallel) combination of motion constraints is the sum of the twist (wrench) spaces of the composing constraints. Analogously, the twist (wrench) space of the parallel (serial) combination of motion constraints is the intersection of the twist (wrench) spaces of the composing constraints. It is important to point out that the reciprocity relation remains valid under serial and parallel combination of motion constraints.

These considerations can be re-formulated in the language of the Grassmann-Cayley algebra. From properties (2) and (4) of the join and meet operators it follows that:

- The twist (wrench) space of the serial (parallel) connection of motion constraints is the support of 


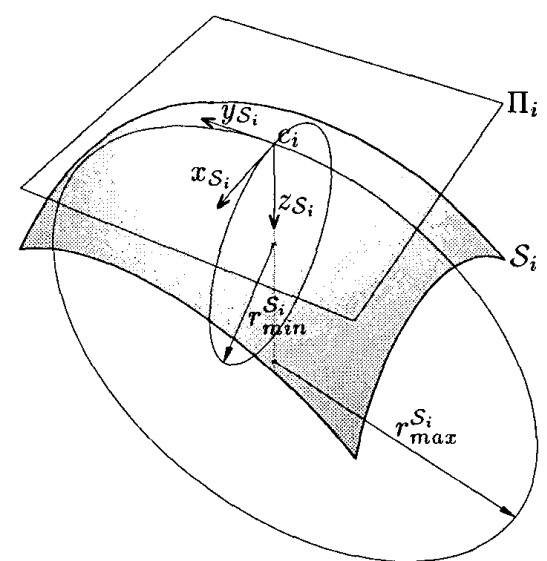

Figure 1: The principal frame of the smooth surface $\mathcal{S}_{i}$ at point $c_{i}$

the join of the extensors that represent the twist (wrench) spaces of the composing constraints, provided that their twist (wrench) extensors are linearly independent.

- The twist (wrench) space of the parallel (serial) connection of motion contraints is the support of the meet of the extensors that represent the twist (wrench) spaces of the composing constraints, provided that the sum of the composing twist (wrench) spaces spans $H\left(H^{*}\right)$.

This considerations will be applied to the study of the kinematic chains that instantaneously are equivalent to the contact between $M$ and $S$.

\section{A Kinematic Model of Point Contacts}

The maximum and minimum curvature directions at a point on a smooth surface are always orthogonal [2] and can be used to define a reference frame called the principal frame (Fig. 1) in which the $x$ axis is directed along the direction of maximum curvature, the $y$ axis along the direction of minimum curvature, and the $z$ axis is directed inward. These axes are uniquely defined unless the point is an umbilic, that is, a point in which the curvature is the same in all directions.

Let us suppose that $M$ and $S$ are in contact at point $c_{i}$ and that their boundaries can be represented, in a neighbourhood of this point, by two smooth surface patches $\mathcal{M}_{i}$ and $\mathcal{S}_{i}$. Let $\Pi_{i}$ be the plane tangent to both surfaces at $c_{i}$. Since it has been assumed that there is no friction between $M$ and $S$, the situation can be kinematically modelled with the kinematic chain represented in Fig. 2 that instantaneously preserves the contact and gives the same motion freedom as the physical contact. Such a motion can be thought as the composition of a slip motion of $M$ in which the contact point does not move with respect to $\mathcal{S}_{i}$, a rotation of $M$ that do not change the contact point with respect both $\mathcal{S}_{i}$ and $\mathcal{M}_{i}$ and, finally, a slide motion of $M$ in which the contact point do not move over $\mathcal{M}_{i}$ [4].

The slip joint can be kinematically modelled by two revolute joints, $R_{\min }^{\mathcal{S}_{i}}$ and $R_{m a x}^{\mathcal{S}_{i}}$, located at the centers of minimum and maximum curvature of $\mathcal{S}_{i}$ whose axes are parallel to the direction of maximum and minimum curvature of $\mathcal{S}_{i}$, respectively.

The rotation of $M$ can be modelled with a revolute joint $R^{\mathcal{S}_{i}, \mathcal{M}_{i}}$ whose axis is normal to plane $\Pi_{i}$ and the slide joint can be represented by means of two revolute joints, $R_{m i n}^{\mathcal{M}_{i}}$ and $R_{m a x}^{\mathcal{M}_{i}}$, located at the centers of minimum and maximum curvature of the surface $\mathcal{M}_{i}$ that have axes parallel to the directions of maximum and minimum curvature of $\mathcal{M}_{i}$, respectively.

In order to model the resulting kinematic chain using the elements of the Grassmann-Cayley Algebra, let us first define a reference frame, $F_{i}$, at the contact point $c_{i}$ such that:

$$
\begin{gathered}
x_{F_{i}}=\left(\begin{array}{cccc}
\cos (\alpha) & -\sin (\alpha) & 0 & 0 \\
\sin (\alpha) & \cos (\alpha) & 0 & 0 \\
0 & 0 & 1 & 0 \\
0 & 0 & 0 & 1
\end{array}\right) x_{\mathcal{M}_{i}, \text { and }} \\
x_{F_{i}}=\left(\begin{array}{cccc}
1 & 0 & 0 & 0 \\
0 & -1 & 0 & 0 \\
0 & 0 & -1 & 0 \\
0 & 0 & 0 & 1
\end{array}\right) x_{\mathcal{S}_{i}}
\end{gathered}
$$

are the transformations relating the principal frames of both surfaces and $F_{i}$.

The center of motion of $R_{m i n}^{\mathcal{S}_{i}}$ is the axis parallel to $x_{\mathcal{S}_{i}}$ passing through the point $\left(x_{\mathcal{S}_{i}}, y_{\mathcal{S}_{i}}, z_{\mathcal{S}_{i}}, 1\right)=$ $\left(0,0, r_{\max }^{S_{i}}, 1\right)$ which, when expressed in the reference frame $F_{i}$, leads to the following extensor:

$L_{\min }^{s_{i}}=\vee\left(\begin{array}{cccc}0 & 0 & -r_{\max }^{\mathcal{S}_{i}} & 1 \\ -1 & 0 & -r_{\max }^{\mathcal{S}_{i}} & 1\end{array}\right)=\left(-1,0,0,0, r_{\max }^{\mathcal{S}_{i}}, 0\right)$.

The center of motion of $R_{\text {max }}^{\mathcal{S}_{i}}$ is the axis parallel to $y_{\mathcal{S}_{i}}$ passing through the point $\left(x_{\mathcal{S}_{i}}, y_{\mathcal{S}_{i}}, z_{\mathcal{S}_{i}}, 1\right)=$ $\left(0,0, r_{\min }^{\mathcal{S}_{i}}, 1\right)$. The corresponding extensor is:

$L_{\max }^{\mathcal{S}_{i}}=\vee\left(\begin{array}{cccc}0 & 0 & -r_{\min }^{S_{i}} & 1 \\ 0 & -1 & -r_{\min }^{S_{i}} & 1\end{array}\right)=\left(0,-1,0,-r_{\min }^{\mathcal{S}_{i}}, 0,0\right)$ 


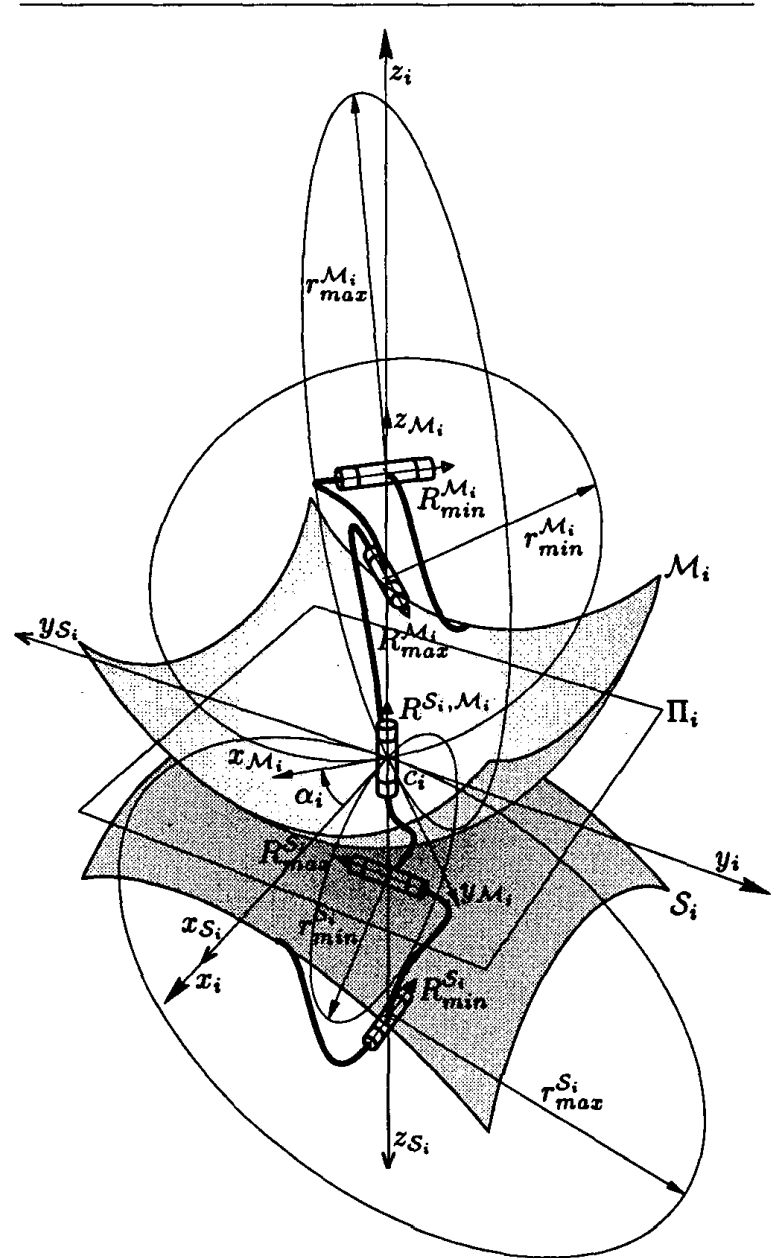

Figure 2: Kinematic model of a surface-surface contact

The center of motion of $R^{\mathcal{S}_{i}, \mathcal{M}_{i}}$ is the axis parallel to $z_{\mathcal{M}_{i}}$ passing trough the point $\left(x_{\mathcal{M}_{i}}, y_{\mathcal{M}_{i}}, z_{\mathcal{M}_{i}}, 1\right)=$ $(0,0,0,1)$. The corresponding extensor is

$$
L^{\mathcal{S}_{i}, \mathcal{M}_{i}}=\vee\left(\begin{array}{llll}
0 & 0 & 0 & 1 \\
0 & 0 & 1 & 1
\end{array}\right)=(0,0,1,0,0,0)
$$

The center of motion of $R_{m a x}^{\mathcal{M}_{i}}$ is an axis parallel to $y_{\mathcal{M}_{i}}$ passing through the point $\left(x_{\mathcal{M}_{i}}, y_{\mathcal{M}_{i}}, z_{\mathcal{M}_{i}}, 1\right)=$ $\left(0,0, r_{\min }^{\mathcal{M}_{i}}, 1\right)$ that, when expressed in the reference frame $F_{i}$, corresponds to an axis having the direction of the vector $\left(-\sin \left(\alpha_{i}\right), \cos \left(\alpha_{i}\right), 0,1\right)$ and passing through the point $\left(x_{F_{i}}, y_{F_{i}}, z_{F_{i}}, 1\right)=\left(0,0, r_{\text {min }}^{\mathcal{M}_{i}}, 1\right)$. The corresponding extensor is:

$$
\begin{aligned}
& L_{\max }^{\mathcal{M}_{i}}=\vee\left(\begin{array}{cccc}
0 & 0 & r_{m i n}^{\mathcal{M}_{i}} & 1 \\
-\sin \left(\alpha_{i}\right) & \cos \left(\alpha_{i}\right) & r_{\min }^{\mathcal{M}_{i}} & 1
\end{array}\right)= \\
& \left(-\sin \left(\alpha_{i}\right), \cos \left(\alpha_{i}\right), 0,-r_{m i n}^{\mathcal{M}_{i}} \cos \left(\alpha_{i}\right),-r_{m i n}^{\mathcal{M}_{i}} \sin \left(\alpha_{i}\right), 0\right) \text {. }
\end{aligned}
$$

The center of motion of $R_{m i n}^{\mathcal{M}_{i}}$ is an axis parallel to $x_{\mathcal{M}}$ passing through the point $\left(x_{\mathcal{M}_{i}}, y_{\mathcal{M}_{i}}, z_{\mathcal{M}_{i}}, 1\right)=$ $\left(0,0, r_{\text {max }}^{\mathcal{M}_{i}}, 1\right)$ that, when expressed in the reference frame $F_{i}$, corresponds to an axis having the direction of the vector $\left(-\cos \left(\alpha_{i}\right),-\sin \left(\alpha_{i}\right), 0,1\right)$ and passing through the point $\left(x_{F_{i}}, y_{F_{i}}, z_{F_{i}}, 1\right)=\left(0,0, r_{\max }^{\mathcal{M}_{i}}, 1\right)$. The corresponding extensor is:

$$
\begin{gathered}
L_{\min }^{\mathcal{M}_{i}}=\vee\left(\begin{array}{cccc}
0 & 0 & r_{m a x}^{\mathcal{M}_{i}} & 1 \\
-\cos \left(\alpha_{i}\right) & -\sin \left(\alpha_{i}\right) & r_{\max }^{\mathcal{M}_{i}} & 1
\end{array}\right)= \\
\left(-\cos \left(\alpha_{i}\right),-\sin \left(\alpha_{i}\right), 0, r_{\max }^{\mathcal{M}_{i}} \sin \left(\alpha_{i}\right),-r_{\max }^{\mathcal{M}_{i}} \cos \left(\alpha_{i}\right), 0\right) .
\end{gathered}
$$

If in the above contact model $r_{\text {max }}^{S_{i}}$ is set to $\infty, R_{m i n}^{\mathcal{S}_{i}}$ becomes a translational joint, i.e. a rotation at infinity, with axis parallel to $x_{\mathcal{S}_{i}}$. Using the points at infinity $\left(x_{\mathcal{S}_{i}}, y_{\mathcal{S}_{i}}, z_{\mathcal{S}_{i}}, 0\right)=(0,0,1,0)$ and $\left(x_{\mathcal{S}_{i}}, y_{\mathcal{S}_{i}}, z_{\mathcal{S}_{i}}, 0\right)=$ $(1,0,1,0)$, the corresponding center of motion can be expressed in the frame $F_{i}$ by the following extensor

$$
L_{\min }^{\mathcal{S}_{i}}=\vee\left(\begin{array}{llll}
0 & 0 & -1 & 0 \\
1 & 0 & -1 & 0
\end{array}\right)=(0,0,0,0,1,0)
$$

Thus, the presented model is general enough to represent, for example, the edge-edge or vertex-face contacts arising when dealing with polyhedra.

\section{Multiple Contacts}

When $M$ keeps several contacts with $S$, a kinematic chain for each contact must be introduced. The extensors have to be referred to the same reference frame, say $F_{0}$, and the resulting parallel mechanism can be analyzed using the join and meet operators.

If the extensors $L_{\text {min }}^{\mathcal{S}_{i}}, L_{\text {max }}^{\mathcal{S}_{i}}, L^{\mathcal{S}_{i}, \mathcal{M}_{i}}, L_{\text {max }}^{\mathcal{M}_{i}}, L_{\text {min }}^{\mathcal{M}_{i}}$ are linearly independent, the twist space $\mathbf{T}_{i}$ of the link $i$ is the support of the 5-extensor $L_{i}=L_{\text {min }}^{\mathcal{S}_{i}} \vee L_{\max }^{\mathcal{S}_{i}} \vee$ $L^{\mathcal{S}_{i}, \mathcal{M}_{i}} \vee L_{\max }^{\mathcal{M}_{i}} \vee L_{\min }^{\mathcal{M}_{i}}$ of $H^{(5)}$, that is,

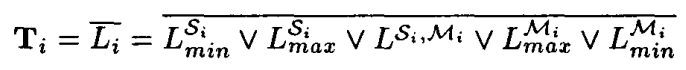

where $L_{m i n}^{\mathcal{S}_{i}}, L_{m a x}^{\mathcal{S}_{i}}, L^{\mathcal{S}_{i}, \mathcal{M}_{i}}, L_{m a x}^{\mathcal{M}_{i}}$ and $L_{m i n}^{\mathcal{M}_{i}}$ are vectors of $H$.

The wrench space $\mathbf{W}_{i}$ of the link $i$ is its dual, that is, the support of the vector $L_{i}^{*}=\left(L_{\min }^{\mathcal{S}_{i}} \vee L_{m a x}^{\mathcal{S}_{i}} \vee L^{\mathcal{S}_{i}, \mathcal{M}_{i} \vee}\right.$ 


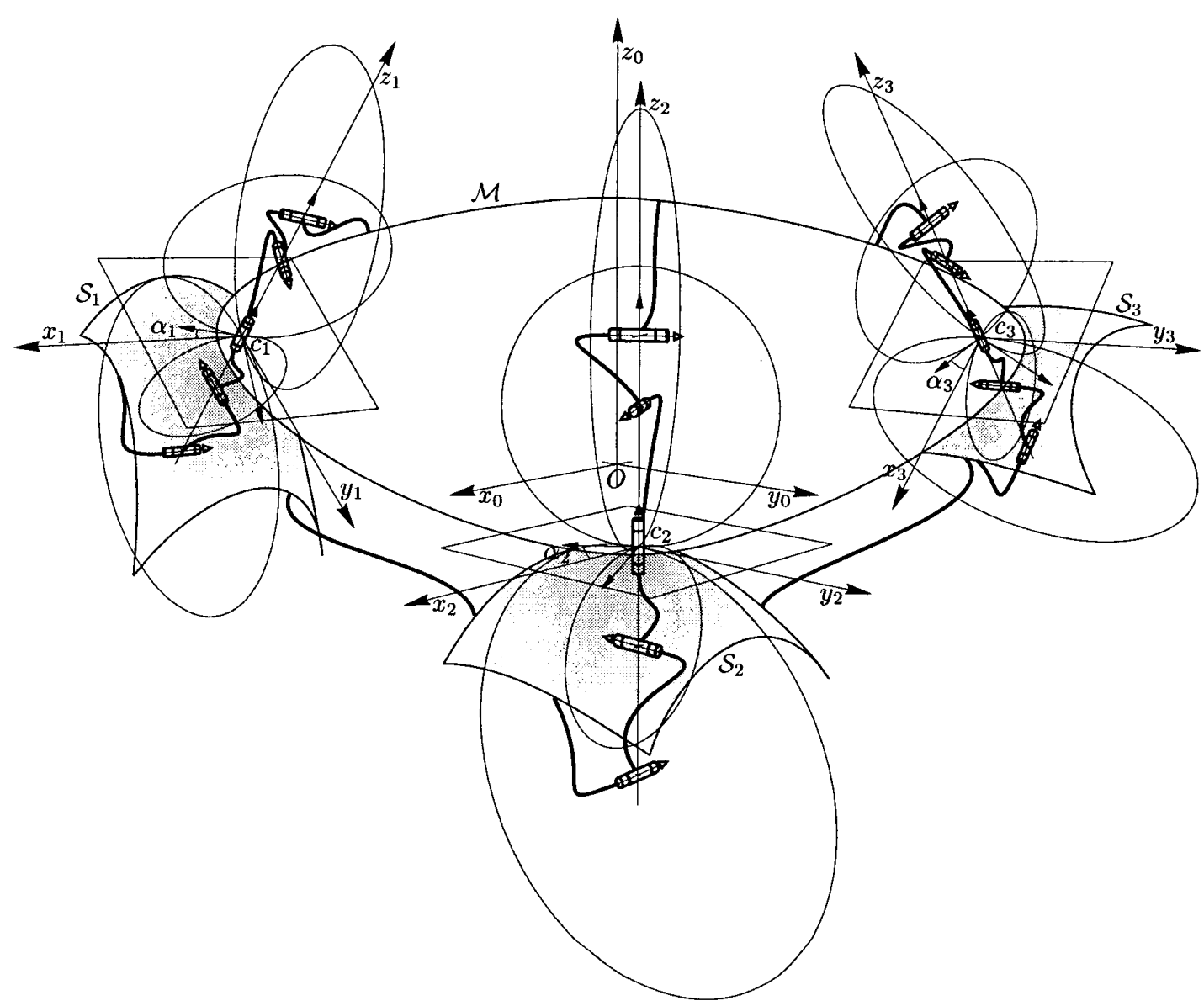

Figure 3: Three contacts between $M$ and $S$ and the instantaneously equivalent kinematic chain

$\left.L_{\max }^{\mathcal{M}_{i}} \vee L_{\min }^{\mathcal{M}_{i}}\right)^{*}=L_{\min }^{\mathcal{S}_{i} *} \wedge L_{\max }^{\mathcal{S}_{i} *} \wedge L^{\mathcal{S}_{i}, \mathcal{M}_{i}^{*} *} \wedge L_{\max }^{\mathcal{M}_{i} *} \wedge L_{m_{i n}}^{\mathcal{M}_{i} *}$ of $H^{*}$, that is,

$$
\mathbf{W}_{i}=\overline{L_{i}^{*}}=\overline{L_{\min }^{S_{i}^{*}} \wedge L_{\max }^{\mathcal{S}_{i} *} \wedge L^{\mathcal{S}_{i}, \mathcal{M}_{i}^{*}} \wedge L_{\max }^{\mathcal{M}_{i}^{*}} \wedge L_{\min }^{\mathcal{M}_{i}{ }^{*}}}
$$

where the extensors $L_{\min }^{\mathcal{S}_{i} *}, L_{\max }^{\mathcal{S}_{i} *}, L^{\mathcal{S}_{i}, \mathcal{M}_{i}^{*}}, L_{\max }^{\mathcal{M}_{i} *}$ and $L_{\text {min }}^{\mathcal{M}_{i}{ }^{*}}$ are 5-extensors of $H^{*(5)}$.

Suppose that $M$ and $S$ make $k \leq 6$ contacts and let $L_{1}, L_{2}, \ldots, L_{k}$ be the extensors of the kinematic chains that are instantaneously equivalent to each one of them.

The twist space $\mathbf{T}$ of $M$ constrained to keep the established contacts with $S$ is the support of the extensor $L=L_{1} \wedge L_{2} \wedge \cdots \wedge L_{k}$, that is,

$$
\mathbf{T}=\bar{L}=\overline{L_{1} \wedge L_{2} \wedge \cdots \wedge L_{k}} .
$$

Since $M$ can apply forces to $S$ only in one direction, the space $\mathbf{W}$ of possible wrenches between $M$ and $S$ does not coincide with the wrench space of its instantaneously equivalent kinematic chain. It is a subspace of the support of the extensor $L^{*}=L_{1}^{*} \mathrm{~V}$ $L_{2}^{*} \vee \cdots \vee L_{k}^{*}$, that is,

$$
\mathbf{W} \subset \overline{L^{*}}=\overline{L_{1}^{*} \vee L_{2}^{*} \vee \cdots \vee L_{k}^{*}} .
$$

It is easy to take this fact into account by appropriately choosing the signs of the coefficients of the linear combination of the wrench extensors in (5).

\section{An Example}

Let us consider the situation represented in Fig. 3 in which the moving body $M$ makes three point contacts with the static environment $S$. 
The twist space $\mathbf{T}$ of the parallel mechanism is the support of $L=L_{1} \wedge L_{2} \wedge L_{3}$. The meet operator is associative, thus we can compute first the meet $L^{\prime}$ between $L_{1}$ and $L_{2}$ and then the meet of $L^{\prime}$ with $L_{3}$. The shuffle formula gives

$$
\begin{aligned}
& L^{\prime}=L_{1} \wedge L_{2}= \\
& +\left[L_{\min }^{S_{1}} L_{\min }^{\mathcal{S}_{2}} L_{\max }^{\mathcal{S}_{2}} L^{\mathcal{S}_{2}, \mathcal{M}_{2}} L_{\max }^{\mathcal{M}_{2}} L_{\min }^{\mathcal{M}_{2}}\right] L_{\max }^{S_{1}} \vee L^{\mathcal{S}_{1}, \mathcal{M}_{1}} \vee L_{\max }^{\mathcal{M}_{2}} \vee L_{\min }^{\mathcal{M}_{1}} \\
& -\left[L_{\max }^{S_{1}} L_{\min }^{\mathcal{S}_{2}} L_{\max }^{S_{2}} L^{\mathcal{S}_{2}, \mathcal{M}_{2}} L_{\max }^{\mathcal{M}_{2}} L_{m i n}^{\mathcal{M}_{2}}\right] L_{\min }^{\mathcal{S}_{1}} \vee L^{\mathcal{S}_{1}, \mathcal{M}_{1}} \vee L_{\max }^{\mathcal{M}_{1}} \vee L_{\text {min }}^{\mathcal{M}_{1}} \\
& +\left[L^{S_{1}, \mathcal{M}_{1}} L_{\min }^{S_{2}} L_{\text {max }}^{S_{2}} L^{S_{2}, \mathcal{M}_{2}} L_{\text {max }}^{\mathcal{M}_{2}} L_{\text {min }}^{\mathcal{M}_{2}}\right] L_{\text {min }}^{S_{1}} \vee L_{\text {max }}^{S_{1}} \vee L_{\text {max }}^{\mathcal{M}_{1}} \vee L_{\text {min }}^{\mathcal{M}_{1}} \\
& -\left[L_{\max }^{\mathcal{M}_{1}} L_{m i n}^{S_{2}} L_{m a x}^{S_{2}} L^{S_{2}, \mathcal{M}_{2}} L_{\max }^{\mathcal{M}_{2}} L_{m i n}^{\mathcal{M}_{2}}\right] L_{m i n}^{S_{1}} \vee L_{m a x}^{S_{1}} \vee L^{S_{1}, \mathcal{M}_{1}} \vee L_{m i n}^{\mathcal{M}_{1}} \\
& +\left[L_{\min }^{\mathcal{M}_{1}} L_{\min }^{S_{2}} L_{\max }^{S_{2}} L^{S_{2}, \mathcal{M}_{2}} L_{m a x}^{\mathcal{M}_{2}} L_{m i n}^{\mathcal{M}_{2}}\right] L_{m i n}^{S_{1}} \vee L_{m a x}^{S_{1}} \vee L^{S_{1}, \mathcal{M}_{1}} \vee L_{m a x}^{\mathcal{M}_{1}} \\
& =\rho_{1} L_{1}^{\prime}+\rho_{2} L_{2}^{\prime}+\rho_{3} L_{3}^{\prime}+\rho_{4} L_{4}^{\prime}+\rho_{5} L_{5}^{\prime},
\end{aligned}
$$

where $L_{i}^{\prime}$ is the $i$-th 4 -extensor of the above expression and $\rho_{i}$ is the value of the corresponding determinant. Since the meet operation is distributive over addition we can write $L=L^{\prime} \wedge L_{3}=\rho_{1} L_{1}^{\prime} \wedge L_{3}+\rho_{2} L_{2}^{\prime} \wedge L_{3}+$ $\rho_{3} L_{3}^{\prime} \wedge L_{3}+\rho_{4} L_{4}^{\prime} \wedge L_{3}+\rho_{5} L_{5}^{\prime} \wedge L_{3}$ where, for example,

$L_{1}^{\prime} \wedge L_{3}=$

$+\left[L_{m a x}^{\mathcal{S}_{1}} L_{\text {min }}^{\mathcal{S}_{3}} L_{\max }^{\mathcal{S}_{3}} L^{\mathcal{S}_{3}, \mathcal{M}_{3}} L_{m a x}^{\mathcal{M}_{3}} L_{m i n}^{\mathcal{M}_{3}}\right] L^{\mathcal{S}_{1}, \mathcal{M}_{1}} \vee L_{m a x}^{\mathcal{M}_{1}} \vee L_{m i n}^{\mathcal{M}_{1}}$

- $\left[L^{\mathcal{S}_{1}, \mathcal{M}_{1}} L_{m i n}^{\mathcal{S}_{3}} L_{m a x}^{\mathcal{S}_{3}} L^{\mathcal{S}_{3}, \mathcal{M}_{3}} L_{\max }^{\mathcal{M}_{3}} L_{m i n}^{\mathcal{M}_{3}}\right] L_{m a x}^{\mathcal{S}_{1}} \vee L_{m a x}^{\mathcal{M}_{1}} \vee L_{m i n}^{\mathcal{M}_{1}}$

$+\left[L_{m a x}^{\mathcal{M}_{1}} L_{m i n}^{\mathcal{S}_{3}} L_{m a x}^{\mathcal{S}_{3}} L^{\mathcal{S}_{3}, \mathcal{M}_{3}} L_{m a x}^{\mathcal{M}_{3}} L_{m i n}^{\mathcal{M}_{3}}\right] L_{m a x}^{\mathcal{S}_{1}} \vee L^{\mathcal{S}_{1}, \mathcal{M}_{1}} \vee L_{m i n}^{\mathcal{M}_{1}}$

$-\left[L_{m i n}^{\mathcal{M}_{1}} L_{m i n}^{\mathcal{S}_{3}} L_{m a x}^{\mathcal{S}_{3}} L^{\mathcal{S}_{3}, \mathcal{M}_{3}} L_{m a x}^{\mathcal{M}_{3}} L_{m i n}^{\mathcal{M}_{3}}\right] L_{m a x}^{\mathcal{S}_{1}} \vee L^{\mathcal{S}_{1}, \mathcal{M}_{1}} \vee L_{m a x}^{\mathcal{M}_{1}}$

The meet between $L_{i}^{\prime}, i=2, \ldots, 5$ and $L_{3}$ can be computed in a similar way.

The space $\mathbf{W}$ of possible wrenches between $M$ and $S$ is a subspace of the support of the 3-extensor $L^{*}=$ $L_{1}^{*} \vee L_{2}^{*} \vee L_{3}^{*}$.

\section{Conclusions}

In this paper a new framework for the analysis of frictionless rigid body interactions has been presented. It is based on the Grassmann-Cayley Algebra by means of which the kinestatic properties of the parallel mechanism resulting from the substitution of each contact by its instantaneously equivalent second order approximation have been studied.

It has been shown that the structure of this algebra fits perfectly to the kinestatic analysis of rigid body interactions due to the kinematic interpretation of the concept of extensor, the existence of an explicit formula for the meet operator and the presence of an intrinsic duality that corresponds to the reciprocity condition between twist and wrench spaces of partially constrained rigid bodies.

This analysis has been carried out with the implicit hypothesis of the absence of uncertainties in the geometric models of the objects involved in the motion but it is also possible to use more general frameworks that include the Grassmann-Cayley Algebra as a subalgebra such as the Grassmann Algebra and the Clifford Algebra. Since these algebras are endowed with a metrics, by means of them it is also possible to deal with geometric uncertainties.

\section{References}

[1] E. Staffetti, L. Ros, and F. Thomas, "Finding infinitesimal motions of objects in assemblies using Grassmann-Cayley algebra," in Proc. of the Tenth World Congress on the Theory of Machines and Mechanisms, pp. 584-591, 1999.

[2] B. O. Neill, Elementary Differential Geometry. Academic Press, 1966.

[3] J.-P. Thirion and A. Gourdon, "The marching lines algorithm: new nesults and proofs," Rapport de Recherche 1881, INRIA, April 1993.

[4] H. Bruyninckx, S. Demey, S. Dutré, and J. De Schutter, "Kinematic models for model-based compliant motion in the presence of uncertainty," The International Journal of Robotics Research, vol. 14, no. 5, pp. $465-482,1995$.

[5] E. Staffetti and F. Thomas, "Kinestatic analysis of serial and parallel robot manipulators using Grassmann-Cayley algebra," in Advances in Robot Kinematics (J. Lenarčič and M. M. Stanišić, eds.), Kluwer Academic Publishers, 2000.

[6] M. Barnabei, A. Brini, and G.-C. Rota, "On the exterior calculus of invariant theory," Journal of Algebra, vol. 96, pp. 120-160, 1985.

[7] N. White, "A tutorial on Grassmann-Cayley algebra," in Invariant Methods in Discrete and Computational Geometry (N. White, ed.), pp. 93-106, Kluwer Academic Publisher, 1995.

[8] N. White, "Grassmann-Cayley algebra and robotics," Journal of Intelligent Robotics Systems, vol. 11, pp. 91-107, 1994.

[9] N. White, "Geometric applications of the GrassmannCayley algebra," in Handbook of Discrete and Computational Geometry (J. E. Goodman and J. O'Rourke, eds.), CRC Press, 1997.

[10] R. S. Ball, A Treatise on the Theory of Screws. Cambridge University Press, 1900.

[11] K. H. Hunt, Kinematic Geometry of Mechanisms. Oxford Science Publications, 1990.

[12] J. Duffy, "The fallacy of modern hybrid control theory that is based on "orthogonal complements" of twist and wrench spaces," Journal of Robotic Systems, vol. 7, no. 2, pp. 139-144, 1990. 